\title{
El derecho al desarrollo pleno: por una oferta programática de los servicios públicos que garantice la atención integral a jóvenes menores de 18 años que presentan consumo problemático de drogas
}

\author{
Joselyn Riffo Chavarría ${ }^{1}$
}

\begin{abstract}
El siguiente artículo analiza el contexto territorial en el que se encuentran insertos jóvenes, de entre 12 y 18 años, de sectores empobrecidos, en cuanto a la actual oferta programática de los servicios públicos para atender el consumo problemático de drogas (licitas e ilícitas). Se indaga en la composición individual, familiar y social de dicha realidad, con el fin de observar los factores que impactan y provocan prácticas abusivas a edades tempranas. Finalmente, se expone de manera crítica el funcionamiento de las redes de intervención y, desde ahí, se plantea la necesidad de contar con mayor oferta especializada, que proteja de forma integral el desarrollo biopsicosocial de la juventud, una garantía que está estipulada dentro la Convención de los Derechos del Niño y ratificada por Chile.

Palabras clave: juventud, vulnerabilidad social, consumo de drogas, oferta programática.
\end{abstract}

The Right to Full Development: A Programmatic Offer of Public Services to Guarantee Comprehensive Care for Minors with Drug Abuse Problems

ABSTRACT

The following article analyzes the territorial context of impoverished youth between 12 and 18 years of age in terms of the public services programs currently offered to address both legal and illegal substance abuse. From there, the individual, family and social composition of this reality is

Chilena. Licenciada en Trabajo Social. E-mail: joo.chavarria@gmail.com 
El derecho al desarrollo pleno: por una oferta programática de los servicios públicos que garantice la atención integral a jóvenes menores de 18 años... / Riffo

investigated in order to identify the factors that impact and provoke abusive practices at an early age. Finally, the article offers a critical look at how the intervention networks operate and, based on this, proposes the need from a more specialized offer to protect the comprehensive biological and psychosocial development of youth, as stipulated in the Convention of the Rights of the Child and ratified by Chile.

Keywords: youth, social vulnerability, drug abuse, programmatic offer.

\section{O direito ao desenvolvimento pleno: por uma oferta programática dos serviços públicos que garanta o atendimento integral a jovens menores de 18 anos que apresentem consumo problemático de drogas}

RESUMO

O seguinte artigo analisa o contexto territorial em que se encontram inseridos jovens, entre 12 e 18 anos, de setores empobrecidos, quanto a atual oferta programática dos serviços públicos para atender o consumo problemático de drogas (licitas e ilícitas). Indaga-se sobre a composição individual, familiar e social desta realidade, com o objetivo de observar os fatores que apresentam impacto e provocam práticas abusivas em idades precoces. Finalmente, o funcionamento das redes de intervenção é exposto de maneira crítica e, desde aí, considera-se a necessidade de contar com maior oferta especializada, que proteja de forma integral o desenvolvimento biopsicossocial da juventude, uma garantia contemplada na Convenção sobre os Direitos da Criança e ratificada pelo Chile.

Palavras-chave: juventude, vulnerabilidade social, consumo de drogas, oferta programática.

\section{Introducción}

La garantía del derecho al desarrollo pleno de niños, niñas y jóvenes es fundamental para ejercer una vida adecuada, coexistiendo como un elemento contemplado en la Declaración Universal de los Derechos Humanos ${ }^{2}$, una base para toda persona.

La Convención de los Derechos del Niño fue suscrita por Chile el año 1990, por lo tanto, el Estado debiera contar con políticas públicas a la altura de dicho tratado internacional, reconociendo

2 Más Información en https://bibliotecadigital.indh.cl/bitstream/handle/ 123456789/903/ declaracion-der.pdf?sequence $=1$ 
y asegurando a toda niñez y juventud, sin distinción, ser beneficiaria

... de una serie de medidas especiales de protección y asistencia; tengan acceso a servicios como la educación y la atención de la salud; puedan desarrollar plenamente sus personalidades, habilidades y talentos; crezcan en un ambiente de felicidad, amor y comprensión; y reciban información sobre la manera en que pueden alcanzar sus derechos y participar en el proceso de una forma accesible y activa. (UNICEF, 1990)

En efecto, uno de los principales desafíos hoy es el sistema de protección integral a la niñez y juventud, el cual se encuentra en tramitación en el Congreso de Chile. Al presente, todavía no existe una ley que dé obligatoriedad a todos los actores sociales en garantizar los derechos de la población infanto-juvenil. Si bien existen en nuestro país sistemas intersectoriales que intentan dar una atención integral a niños, niñas y sus familias, como por ejemplo la creación de la política pública de Chile Crece Contigo ${ }^{3}$, esta no logra llegar al total de la población menor de 18 años, ya que se encuentra orientada a la atención de la primera infancia, hasta el término del primer ciclo básico (alrededor de los 8 o 9 años).

El presente artículo analiza cómo impacta en la juventud empobrecida la temática del consumo problemático de drogas - jóvenes insertos en un contexto social de alta vulnerabilidad social-, mostrando los servicios de protección, tratamiento y de redes que intervienen en un intento de detener situaciones de vulneración. Desde ahí se desprenden las temáticas de las concepciones de juventud; juventud en contexto de alto riesgo social; familias y factores socioeconómicos; pobreza; redes; territorio; políticas públicas; oferta programática y situación actual de la niñez en Chile, desde una lectura crítica para el análisis del sistema de garantes y sus niveles de responsabilidad, en los cuales el planteamiento de

Más Información en http://www.crececontigo.gob.cl/acerca-de-chcc/que-es/ 
exigir una protección integral real para los derechos humanos de toda niñez y juventud es transcendental.

\section{Aproximaciones al concepto de "juventud"}

La etapa de lo juvenil es un momento importante dentro de los cambios del desarrollo progresivo, tanto en aspectos biológicos como psicológicos. Si avanzamos hacia una aproximación al concepto clásico de "juventud”, la Organización Mundial de la Salud (2018) señala que corresponde a un "periodo de crecimiento y desarrollo humano que se produce después de la niñez y antes de la edad adulta, entre los 10 y los 19 años", aunque este concepto hace referencia puntualmente al término de adolescencia, siendo para la OMS un periodo de transición biopsicosocial que sucede entre la infancia y la adultez, con cambios corporales y adaptación a nuevas estructuras psicológicas.

Asimismo, es señalado por los postulados de Erickson (1977) en su descripción de la etapa del desarrollo psicosocial, la cual plantea a la juventud con roles tradicionales, uniformes y homogéneos, definiciones altamente utilizadas en la enseñanza académica, como también en los procesos de investigación y caracterización de lo juvenil por las ciencias sociales (citado en Duarte, 2000).

Al observar dichas definiciones, se aprecia un establecimiento de patrones de comportamientos socialmente esperados para definir determinados periodos de la vida humana, en los cuales los autores citados se refieren a la adolescencia o una sola realidad de juventud y no al concepto de "juventudes", instaurando para ello rangos de edad con características dadas en la conducta, por lo que si una persona no cumple dichos criterios estaría faltando a lo socialmente esperado. Estos criterios, establecidos a través el uso, son una forma de moldeamiento dentro del desarrollo humano en sus diferentes etapas. Sin embargo, se puede constatar que dicho fenómeno no se encuentra dado de forma estricta y universalmente cuando se define a la juventud -o a la niñez o vejez-; más bien, todas estas experiencias de vida se van 
construyendo socialmente (Bourdieu, 1990). Por lo que la edad es un dato utilizado para la manipulación, ya sea a través de la imposición de identidades juveniles por el comercio o la moda, intereses o concepciones que no abordan las pluralidades, más bien universalizan patrones en las diferentes edades biológicas de la vida humana.

El postulado que asume a la juventud como una experiencia de transición y preparación para el mundo adulto se encuentra altamente reflejado en las concepciones ya mencionadas de Erickson (1998), quien menciona dentro de la experiencia de la juventud el aspecto de "identidad profesional" que una persona debería vivenciar, haciendo alusión a la experiencia académica universitaria como una forma de preparación para la adultez y el sistema laboral, es decir, un proceso que se experimenta dentro de la juventud (citado en Bordignon, 2005). Esta descripción de lo juvenil, de parte del autor, se refiere a un contexto específico, o sea, la juventud que puede acceder a dicha realidad universitaria, invisibilizando las distintas juventudes existentes en la población mundial, las cuales no pueden experimentar dicha posibilidad académica, ya sea por factores religiosos, sociales, culturales y/o económicos, siendo una teoría poco realista e inclusiva para el presente análisis.

Esta forma de interpretación de la juventud hacia la adultez estará dada solo por el nivel participación que un joven tiene dentro del mercado del consumo y la producción, dejando fuera a quienes no optan por dicha forma de vida o, simplemente, a quienes no pueden acceder a ella por las escasas posibilidad que el mundo social les entrega.

El contexto sociohistórico por el cual transitan las juventudes de una época determinada impacta en la construcción de lo juvenil y en la manifestación de los comportamientos; en dicho contexto, el joven es influenciado por las tendencias existentes desde el modelo de vida imperante, el cual moldea y/o manipula las necesidades materiales y sociales. 
Por ello, resulta fundamental tener una real comprensión de los fenómenos sociales estudiados, en este caso de la juventud empobrecida en contexto de alta vulnerabilidad, de 12 a 18 años de edad, con el fin de analizar de forma correcta los diversos factores, socioculturales o económicos, que impactan en los intereses de la población y en sus formas de vida, rompiendo la mirada tradicionalista actual, que surge bajo un contexto territorial e histórico específico, y, de esta forma, reconocer su pluralidad y heterogeneidad al momento de su estudio.

Se trata de plantear a las juventudes desde sus diversas realidades e historias de vida, reconociendo a los jóvenes como sujetos con necesidades básicas y otras específicas de acuerdo con el entorno donde crecen. La juventud es un momento pleno de transformaciones fisiológicas, que se experimentan en un territorio determinado por factores históricos, económicos y socioculturales (Rodríguez, 2005).

\section{Juventud en contexto de alta vulnerabilidad social}

La comprensión analítica del contexto en los cuales nacen, se desarrollan y viven las juventudes del presente estudio descriptivo es fundamental, con el fin de contar con elementos que permitan visualizar el sujeto en cuestión y sus posibles problemáticas sociales que surgen a partir del territorio en que habitan y los servicios públicos existentes para abordar dicha realidad social.

Un contexto que presenta alta vulnerabilidad social, entendiéndose la vulnerabilidad como "un desajuste entre los activos y la estructura de oportunidades, es decir, los activos serían insuficientes, poco pertinentes o difíciles de manejar para aprovechar la estructura de oportunidades existente" (Rodríguez, 2005, p. 18), ya sean presentados estos como servicios sociales en el ámbito de salud, educación, habitacional, empleo y/o protección. Cuando estos activos no logran llegar a la población destinada o simplemente no existe una oferta dentro de la estructura en la que se vive, se presentan riesgos en el acceso a las posibilidades 
de cumplir un conjunto de condiciones socioeconómicas asignadas a una persona, grupo familiar o población específica, asociadas al bienestar integral para el desarrollo y exigencias de la actual vida.

Si adicionamos a esto, dificultades en cuanto a recursos individuales y familiares, ya sea por precariedad dentro de la historia vital de una persona, sometida a vivencias estresoras al convivir en un ambiente frágil, ello redunda en sentimientos de inestabilidad respecto del futuro y carencias para desarrollar una identidad segura frente al medio social que experimenta.

... la precarización como un proceso en que el sujeto es sometido a presiones y experiencias que lo conducen a vivir una existencia frágil en el presente, sometido a incertidumbres acerca del futuro, con una identidad insegura y carente de un sentido de desarrollo posible por medio del trabajo y el estilo de vida. (Cuevas, 2015, p. 3)

Añadido a lo anterior, el factor "pobreza" aparece como uno de los fenómenos sociales más importantes que afecta la calidad de vida y el bienestar de la población. Así lo menciona el Ministerio de Desarrollo Social (2011), señalando que

la pobreza involucra una serie de privaciones en las familias que la padecen y, generalmente, está asociada a la existencia de otros fenómenos negativos, tales como la desigualdad y la falta de oportunidades. La pobreza es un fenómeno complejo que admite más de una definición y un tipo de medición. Normalmente, entendemos por pobreza la condición en la cual las personas no tienen los recursos suficientes para satisfacer de manera adecuada sus necesidades básicas.

En consecuencia, es importante considerar el factor "pobreza" para el presente análisis, no solo desde una perspectiva de la insuficiencia de ingresos económicos de un grupo específico, como habitualmente es definida y medida en nuestro país (Ministerio de Desarrollo Social, 2011), más bien, para comprender de mane- 
ra global o multidimensional ${ }^{4}$ la realidad de la juventud estudiada. La pobreza también debe ser pensada desde la vulnerabilidad social, ya que, paralelamente al factor económico, se agregan los aspectos de inseguridad individual y social, indefensión, exposición de riesgos, vulnerabilidad frente al carácter de las estructuras institucionales existentes en un lugar determinado, y el efecto que estos procesos tienen como causales del empobrecimiento de un territorio, grupo o individuo (Pizarro, 2001).

Con ello, la juventud siempre ha sido considerada como un grupo vulnerable, debido a los factores socioeconómicos mencionados, además de variados elementos y condiciones de desprotección (indefensión) frente al escenario social en el cual viven. Estos hechos agudizan más su realidad, necesitando de un abordaje especial en cuanto a políticas públicas destinas a esta población. En este sentido, se debe tener en cuenta, al momento de trabajar y analizar a la juventud, específicamente la del presente estudio (entre 12 a 18 años de edad), las dimensiones que impactan en su condición de vulnerabilidad, tales como los riesgos en la historia vital de un joven, la dimensión institucional en la que están insertos (configurada principalmente desde el adultocentrismo ${ }^{5}$ ), la inserción educativa, de salud, recreativa y económica que la sociedad ofrece a los jóvenes, etc. Todas estas variantes van moldeando el contexto actual, la composición del territorio específico desde el que se configuran hoy las juventudes de realidades sociales empobrecidas (Rodríguez, 2005).

4 Se entenderá como "adultocentrismo" la práctica de autoridad legitimada "naturalmente" por la sociedad y "producida como resultado de diversos procesos económicos y políticos que han consolidado materialmente este modo de organización societal, así como de la construcción de su matriz sociocultural que -en un plano simbólico-, potencia su recreación y reproducción, construyendo imaginarios que afectan directamente las relaciones y los procesos identitarios de diversos sujetos sociales" (Duarte, 2012, p. 105).

5 Cuando se habla de pobreza multidimensional, se está haciendo referencia a la inclusión de las carencias que afectan a las personas y sus familias desde una perspectiva más amplia, al tomar en cuenta indicadores como el entorno y las redes (educación, trabajo, salud, seguridad social, vivienda, cohesión social (Ministerio de Desarrollo Social, 2017, p. 17). 
En ese aspecto, se puede mencionar que, en Chile, la pobreza presenta un mayor índice en los grupos de menores edades. Al desagregar dicho grupo en tramos de edad, se aprecia que, a menor edad, mayor es la incidencia del factor pobreza por ingreso (Ministerio de Desarrollo Social, 2017).

En la actualidad, existen 4.369 .035 personas entre 0 y 17 años en Chile. Este segmento representa a un $24,9 \%$ de la población del país, según la Encuesta $\mathrm{CASEN}^{6}$ 2015. La pobreza en niños, niñas y juventud continúa mostrando tasas altas de pobreza multidimensional y por ingresos en relación con los adultos mayores de 18 años, es decir, que hoy más de 790.000 niños, niñas y adolescentes viven en situación de pobreza por ingresos y más de 980.000 mil en situación de pobreza multidimensional. Aunque también se debe mencionar que los índices de dicha realidad han disminuido con el pasar de los años, como refieren encuestas de años anteriores (CASEN), pero aún es una problemática que subsiste.

En la misma línea, se aprecia que la juventud más desfavorecida, ubicada dentro de los colectivos vulnerables de la sociedad, posee condiciones que la mantienen en escenarios de riesgo, ya por componentes estructurales, o personales o sociales, posicionándolos en un contexto de desventaja. Dicha realidad de desventaja social puede ir acumulándose cuando persisten estas experiencias, llegando a ser detonante en la generación de entornos con escasa integración sociocultural, educativa, laboral o política, llevando a una persona a la exclusión total cuando no se cuenta con redes de apoyo (Bendit y Stokes, 2004).

Dentro de los factores relevantes que determinan las desventajas que experimentan los jóvenes de realidades empobrecidas, los au-

La encuesta de Caracterización Socioeconómica Nacional (CASEN), del Ministerio de Desarrollo Social, es una encuesta a hogares, de carácter multipropósito, es decir, que barca diversos temas como educación, trabajo, ingresos, salud, entre otros; además, es una encuesta transversal, por lo tanto, incluye a todo el espectro de la población del país. 
tores Bendit y Stokes (2004) postulan que la sociología identifica estructuras asociadas a un precario trasfondo familiar, con padres de bajo nivel educativo; grupo familiares con ingresos económicos frágiles; además de inestabilidad laboral, desempleo, bajos recursos sociales, problemáticas habitacionales o viviendas en mal estado e inseguras, necesidades básicas insatisfechas, discriminación en relación al sexo, a oportunidades de formación, estereotipos y expectativas puestas en la juventud desde el mundo adulto; trabajo prematuro en menores de edad, deserción escolar, escasez de apoyo institucional para la juventud (insuficiente política pública), factores culturales transgeneracionales, relaciones interpersonales intrafamiliares dañinas para el positivo desarrollo biopsicosocial de una persona y discursos político-ideológicos con estigmatización de la juventud, desde un enfoque más negativo (delincuencia juvenil/adolescente).

Continuando con el postulado anterior, como factores sociales, también existen factores individuales que cobran gran importancia en la influencia que ejercen sobre las desventajas sociales en la construcción de lo juvenil. En el lugar donde habitan deficiencias estructurales, o sea, donde no hay un soporte social para todos los integrantes de una comunidad, la perspectiva individualista empieza a operar, determinando dichas deficiencias o nulas oportunidades. Es decir, en dicho escenario individualista, se responsabiliza al individuo frente la situación social de desventaja que vive, y no a la estructura social en la que vive y que genera exclusión. Los factores individuales asociados a la desventaja social van ocurriendo cuando existen situaciones familiares problemáticas, rigidez en las relaciones de socialización, falta de redes de ayuda, enfermedades, pobreza, enfermedades de salud mental no tratadas, factores psicológicos como prioridades personales, desmotivación, predominio de conducta inadaptada en escenarios críticos, baja autoestima, carencia de habilidades sociales, etc.

Por ello, aparte del soporte social y estructural que debe tener el contexto social en el que se inserta una persona, resulta funda- 
mental un apoyo familiar, tanto emocional como material, que logre brindar protección en todos los ámbitos a un niño, niña o joven, con el fin de contar con un buen desarrollo biopsicosocial, como lo menciona el artículo $\mathrm{N}^{\circ} 6$ de la Convención de los Derechos del Niño (UNICEF, 1990), tratado al cual Chile está suscrito, haciendo referencia al derecho a la vida, la supervivencia y el desarrollo.

\section{Familias y factores problemáticos}

La familia, según la Declaración Universal de los Derechos Humanos (Asamblea General de las Naciones Unidas, 1948), es el elemento natural y fundamental de la sociedad y tiene derecho a la protección de la sociedad y del Estado. El concepto de "familia" y su composición son los lazos principales de afinidad en el establecimiento de un vínculo reconocido entre sus integrantes.

Artículo 16 de la Declaración Universal de los Derechos Humanos. Asamblea General de las Naciones Unidas: La familia es el elemento natural y fundamental de la sociedad y tiene derecho a la protección de la sociedad y del Estado. (Biblioteca del Congreso Nacional de Chile, 2009)

Todo territorio se compone de individuos y sus grupos familiares vinculares, los cuales se van definiendo de acuerdo con su historia dentro del contexto estructural y sociocultural que habitan. Desde ahí se desarrollan sus habitantes: a partir de las posibilidades que entrega dicha realidad social se van formando y construyendo, mediante el intercambio de saberes y valores transmitidos principalmente por sus figuras de crianza.

La familia es el primer ambiente donde una persona se relaciona, siendo de gran impacto la experiencia que se tiene en ella, la forma en que se entregan mecanismos de socialización hacia un niño, niña y joven para la integración con su entorno.

La juventud y sus familias, pertenecientes a sectores sociales con mayor vulnerabilidad, no siempre cuentan con todos los servicios 
sociales que permitan un grado de desarrollo conveniente para insertarse de manera integral dentro de la sociedad, por lo que se ven limitados en conseguir activos para satisfacer todas sus necesidades respecto de salud, educación, vivienda, protección o desarrollo de herramientas personales.

En muchas ocasiones, el factor económico es frágil, experimentándose sensación de fracaso en este ámbito, desequilibrio entre sus aspiraciones, expectativas y oportunidades que se traducen en desigualdad social. Dichos grupos se ven enjuiciados, al no poder competir con el actual modelo de vida, percibiendo procesos de marginación producto de la asimetría entre las diferentes clases sociales y entrando en un conflicto psicosocial de la experiencia vivida (Comellas, 1996).

Algunos jóvenes aceptan dicha realidad y logran adaptarse dentro de las pocas posibilidades que les presenta su medio, al contar con un mínimo soporte de cualidades personales. Mientras otros utilizan métodos no legitimados por la sociedad para conseguir dichas oportunidades, o rompen con los medios ofrecidos, aislándose socialmente.

El trascurso de una persona para segregarse socialmente y definirse como "multiproblemática" ocurre a partir de diversos factores, dentro de un proceso social dinámico que la misma estructura social consolida, por elementos que favorecen un estado de marginalidad, tales como la desorganización familiar, existencia de carencias emocionales y herramientas de socialización entre sus miembros, elementos que obstaculizan la incorporación de valores y normas sociales dentro de un grupo. No se estimulan valores educativos, construyéndose interpretaciones de la realidad sin un referente adecuado para la inserción y procesos de socialización. La reacción de la sociedad frente a esta población de personas será la discriminación, respuesta reforzada por las creencias transmitidas, ejerciendo apartamiento de los grupos marginados (Comellas, 1996). Por lo tanto, un contexto social que refuerce la marginación, como valores trasmitidos dentro 
del territorio donde se vive, se transforma en un contexto de riesgo desde la estigmatización social que se robustece e impera.

Resulta importante señalar que, al hablar de familias multiproblemáticas, no se debe caer en generalizaciones desde la definición que se presenta en este estudio, ya que existen particularidades en todo territorio. Sin embargo, para dar una explicación sobre su dinámica, se puede mencionar que su nacimiento ocurre dentro de las estructuras sociales mencionadas en los párrafos anteriores, en las cuales los factores sociales y la escasez de servicios sitúan en un escenario de insuficientes medios de cambio para dicha realidad.

Entre las definiciones, se puede puntualizar que las familias multiproblemáticas viven en territorios de alto riesgo social, caracterizados estos por evidentes dificultades económicas (pobreza), carencias materiales, sociales y escasos medios para salir de dicha posición. Zonas en que se aprecian diversas problemáticas, tanto individuales como familiares, en las cuales los miembros que habitan el lugar necesitan la posibilidad de contar con mayores recursos sociales para acceder a un nivel de vida adecuado y desarrollarse de forma sana, como lo menciona la siguiente definición.

La pobreza puede entenderse como una de las formas extendidas de maltrato social estructurado. Es necesario dejar claro que la pobreza no es sinónimo de familia desestructurada o FMP (familia multiproblemática). La falta de medios y de competencias sociales reconocidas, así como las dificultades para cubrir las necesidades diarias y para proporcionar los elementos educativos necesarios, incrementan el riesgo de marginación, la tensión familiar y el estrés individual, facilitando la existencia de comportamientos distorsionados y dificultando la posibilidad de una reacción eficaz delante de su manifestación. Los valores culturales e individuales se desmoronan y se empieza una dinámica circular difícil de romper, basada en la inmediatez de las actividades, que predispone a la negligencia y pasividad social y a la preponderancia de actividades sumergidas para poder garantizar la supervivencia. (Comellas, 1996, p. 4) 
En dichos contextos sociales nacen y se desarrollan muchas juventudes, marcadas en el trascurso de su historia vital por episodios de falta de oportunidades en su cotidiano. Por otro lado, también deben vivenciar problemáticas provenientes desde su grupo familiar. En este sentido, se puede nombrar cuatro ejes descriptivos que presentan las familias con multiproblemas: Polisintomatología y crisis recurrentes; Desorganización; Abandono de las Funciones Parentales, y Aislamiento (Gómez, 2007).

Respecto de los puntos uno y dos que refiere el autor, se relacionan con situaciones de negligencia, consumo problemático de alcohol, violencia intrafamiliar, abuso de sustancias y depresión dentro de la experiencia de vida.

El eje de desorganización en una familia multiproblemática se refiere a la dinámica de caos en las relaciones interpersonales de los miembros y a comunicación disfuncional. Existencia de conflictos, falta y poca claridad de límites, insuficientes reglas y normas, roles difusos, jerarquía caótica, lo que provoca acciones agresivas en integrantes del grupo. Al mismo tiempo, en este aspecto, la comunicación es desorganizada, con información pobre para el intercambio y ambivalencia relacional, la cual está asociada a desórdenes vinculares entre personas del mismo grupo familiar.

La ambivalencia e incongruencia encuadran una dificultad para "leer" adecuadamente los matices e implicancias de un mensaje y para desarrollar empatía en la comunicación. En situaciones de resolución de conflictos, tienden a interpretarse las "señales" comunicativas como muestras de rechazo o abandono. Como el lenguaje ha perdido su capacidad mediadora, se instalan los golpes y gritos, o la autoagresión y autodestrucción, el acting out como solución de "punto final" y de descarga de la tensión acumulada. (Gómez, 2007)

El punto tres, abandono de las funciones parentales, señala que los estudios recientes de las familias multiproblemáticas dan cuenta que abandonan las funciones de marentalidad/parentalidad o existe incompetencia en el ejercicio. Cuando un joven se desarro- 
lla dentro de una familia debilitada en cuanto a la entrega de capacidades socializadoras para la inserción social, sin que se le facilite la internalización de normas y valores imperantes socioculturalmente, inhibe el respeto a la sociedad y entra en un estado de conflicto con su entorno. Este ámbito está ligado a que las funciones parentales/marentales son entregadas a terceros (vecinos, conocidos, familia extensa, profesionales).

El último punto, aislamiento en las familias multiproblemáticas, alude al distanciamiento físico y afectivo, tanto de la familia extensa como del apoyo que podría brindar la red social e institucional del territorio en el que se vive, siendo un factor de alto riesgo, en especial en los procesos de cuidado y crianza. Ocurre cuando, teniendo el joven familia extensa, esta no logra ser un soporte de ayuda, al estar constituida bajo pautas de marentalidad/parentalidad negligente; suele ser una red precaria, con cuidadores que no entregan activos seguros para un positivo desarrollo biopsicosocial de un niño, niña o joven.

Existen pautas dañinas dentro de lo familiar, que se van trasmitiendo de forma transgeneracional, relacionadas con el factor "pobreza" (antecedentes de baja escolaridad, dinámicas disfuncionales en su interior, violencia). Los miembros de las familias que se han mencionado, padre, madre, hermanos mayores de edad, abuelos, tíos, terceros cercanos, poseen inhabilidades sociales que impiden al joven integrarse de manera adecuada dentro de una comunidad, trasmitiendo estas pautas al hacer familia con sus hijos/hijas.

La pobreza emerge como una variable de particular influencia en la cronificación de conductas parentales negligentes (si bien no debe igualarse pobreza a negligencia, ni a las otras características señaladas previamente). Los efectos indirectos de la pobreza que potencian el desarrollo de dinámicas negligentes y/o maltratadoras, incluyen historias de traumatismo y deprivación, condiciones de adversidad crónicas, mayor cantidad de variables de estrés parental, aislamiento social, mono- 
parentalidad, embarazos adolescentes, y vivir en comunidades que son peligrosas, violentas y que carecen del capital social necesario para apoyar la crianza de los hijos. (Gómez, 2007)

Cabe destacar que las familias multiproblemáticas no solo se presentan en contexto de alta vulneración social, con factores de pobreza y depravación sociocultural, también se pueden encontrar en familias con un mayor nivel económico. No obstante, la condición de pobreza agrega un efecto que acentúa los riesgos en las dinámicas de sus miembros, volviéndose relaciones estructuradas muy difícil de cambiar.

Otro elemento a mencionar es que el concepto de "familia multiproblemática", recurrentemente es asimilado como una condición en la cual la familia es la principal responsable de dicha situación, dejando afuera los diversos elementos que componen a una sociedad, como las oportunidades sociales, culturales, el contexto político-estructural, como se ha explicado en el presente estudio, que van impactando también en cómo se hace familia en un contexto especifico, siendo una responsabilidad de todos.

\section{Juventud, sus familias y la vulnerabilidad social}

Se puede analizar que los jóvenes del presente estudio, sus familias y su contexto territorial, se van definiendo por un conjunto de variables, valores ideológicos y características socioeconómicas que se interrelacionan entre sí, configurando de esta forma su realidad y moldeando el actuar de intereses individuales.

En este aspecto, la familia y sus estilos de crianza son de gran importancia dentro del desarrollo social, en especial en el involucramiento que las figuras de cuidado van teniendo frente al desarrollo de sus integrantes. Entre menos involucramiento en el proceso del ciclo vital de un joven, mayor será la probabilidad de que éste enfrente sentimientos de angustia. Al estar expuesto a un ambiente familiar de abandono afectivo y desprotección, las conductas de riesgo son latentes, existiendo posibilidades de prácticas poco sanas a muy temprana edad. 
En consecuencia, cuando existen condiciones ambientales de alta vulnerabilidad social en el lugar donde se habita, sumado a ser parte integrante de una familia que posee diversas problemáticas en sus relaciones sociales, se crean condiciones de alta inseguridad para el desarrollo biopsicosocial de un joven de entre 12 a 18 años, pudiendo padecer sentimientos de indefensión y de abandono de parte de sus figuras de cuidado. Si dichas condiciones familiares se experimentan desde los primeros años de vida y se mantienen en el tiempo, se provocan dificultades en la salud mental de una persona, manifestándose fuertemente en la etapa de "adolescencia”. La prevalencia a que dichos estados de afectación sean compensados mediante el consumo de sustancias es probable, como el alcohol y drogas, ya que la juventud de entre 12 y 18 años se encuentra en un momento de ciclo vital relevante, al estar en pleno desarrollo biológico, personal y social.

Estudios internacionales de los últimos 20 años dan cuenta de que el consumo de drogas en la juventud está ligado a la prevalencia de síntomas depresivos. Este hecho fue corroborado por un estudio que se realizó en 95 colegios de la Región Metropolitana en 2012, con "adolescentes" de 12 a 20 años de edad, en los que la prevalencia de sintomatología depresiva clínicamente fue significativa, varió entre 13,2\%10 y 44\%11, y el tabaco, alcohol y marihuana era lo más consumido por los jóvenes en etapa escolar (Rojas Castillo at al., 2012).

En la misma línea, $\mathrm{SENDA}^{7}$ informa que el alcohol (droga lícita) es la más consumida en Chile (Informativo SENDA, 2017).

La última encuesta realizada por el Instituto Nacional de la Juventud (INJUV, 2015, p. 18) muestra resultados sobre el aumento del consumo de alcohol, LSD y marihuana respecto de 2012, siendo la marihuana la que registra un alza de 17 a $29 \%$.

Servicio Nacional para la Prevención y Rehabilitación del Consumo de Drogas y Alcohol. Informe SENDA 2017. Recuperado de http://www.injuv.gob.cl/storage/ docs/Libro Octava Encuesta Nacional de Juventud.pdf 
Respecto a la edad, si bien el consumo de drogas suele ser declarado con mayor frecuencia por la población de 20 años o más, en las y los adolescentes (de 15 a 19 años) el consumo de marihuana, en particular, presenta diferencias estadísticamente significativas bajas respecto a jóvenes entre 20 y 24 años, y nulas cuando se compara con jóvenes entre 25 y 29 años de edad. El consumo de drogas lícitas también representa un peligro para este grupo, pues los porcentajes de la población que reporta consumo de alcohol y cigarrillo alcanzan cifras que duplican el porcentaje de quienes declaran consumir marihuana ( $57 \%$ y $41 \%$ respectivamente) y en cuanto a las cantidades que consumen, no muestran diferencias estadísticamente significativas con jóvenes de 20 años o más.

En las encuestas mencionadas, se observa que el consumo de drogas lícitas, como el alcohol y el tabaco, en jóvenes en etapa escolar (15 a 19 años) es alto, normalizándose y permitiéndose socialmente. El uso de estas sustancias va en aumento con el paso del tiempo.

Los resultados y datos mostrados por las encuestas deben ser utilizados para su reflexión, porque es necesario contar con un servicio que atienda dicha problemática, ya que entre más temprano se aborde, menor será el costo de su tratamiento para la sociedad. $\mathrm{Al}$ respecto, Shonkoff (2010) afirma que las bases de una sociedad exitosa se construyen en la primera infancia: "el desarrollo sano de los niños más pequeños, provee los cimientos de una adultez sana y competente, de una ciudadanía responsable, de la productividad económica, de comunidades fuertes y de una sociedad justa y sostenible" (citado en Chile Crece Contigo, 2015).

Asimismo, el costo económico de la atención del consumo de drogas, y todo lo asociado a ello, para un país, es alto. Según Fernández (2012), los recursos tanto monetarios como humanos, los costos en prevención, salud, pérdidas de productividad y control del delito - cuando se llega a infringir la ley debido a la necesidad de consumo-, permiten apreciar la noción de costo de oportu- 
nidad, los gastos en que se incurre para atenderlo, cuando estos podrían haber sido utilizados en otras actividad alternativas, potencialmente protectoras y preventivas, disminuyendo el daño y la vulneración de derechos a la población juvenil y niñez.

Es importante destacar que la atención de salud integral del adolescente es aquella que permite evaluar su estado de salud, así como el crecimiento y normal desarrollo, fomentando precozmente los factores y conductas protectoras e identificando precozmente los factores y conductas de riesgo, así como los problemas de salud, con objeto de intervenir en forma oportuna, integrada e integral, con enfoque anticipatorio, participativo y de riesgo. (Ministerio de Salud, 2011)

No obstante, Chile carece de una oferta efectiva en la prevención y tratamiento del consumo de sustancias en jóvenes. Las políticas públicas referentes a drogas se enmarcan en un enfoque prohibicionista más que preventivo. Como efecto de este paradigma, se han implementado una serie de dispositivos de control sanitariopenal, entre ellos el espacio de tratamiento que termina articulándose más como un instrumento educativo y normalizador más que un espacio terapéutico.

La prohibición es arbitraria porque es una doctrina y no una política fundada en datos científicos. Una ideología basada en la represión social que enaltece al hombre adaptado dictaminando lo permitido y no permitido, el bien y el mal, lo sano y lo enfermo. La prohibición es el ámbito del orden y no de salud, de la moral y no de la ciencia. (Vivanco, 2015, p. 81)

Por eso, los actuales programas de rehabilitación para el tratamiento ambulatorio del consumo de sustancias en menores de edad se encuentran enmarcados bajo la oferta ofrecida desde el Servicio Nacional para la Prevención y Rehabilitación del Consumo de Drogas y Alcohol (SENDA, ex $\mathrm{CONACE}^{8}$ ), una entidad 
del gobierno de Chile responsable de elaborar políticas de prevención del consumo de alcohol y otras drogas, así como de tratamiento, rehabilitación e integración social de las personas afectadas por estas sustancias (SENDA, 2011).

SENDA es un servicio radicado en el Ministerio del Interior y Seguridad Pública, creado el 21 de febrero de 2011 por la Ley $\mathrm{N}^{\circ}$ 20.502, que nace a partir de la responsabilidad de elaborar, coordinar, ejecutar y evaluar las políticas públicas destinadas a prevenir la delincuencia y rehabilitar y reinsertar socialmente a los infractores de la ley, SENDA

tendrá como principal misión ser el colaborador directo e inmediato del Presidente de la República en asuntos relativos al orden público y la seguridad pública interior, para cuyos efectos concentrará la decisión política en estas materias, y coordinará, evaluará y controlará la ejecución de planes y programas que desarrollen los demás ministerios y servicios públicos en materia de prevención y control de la delincuencia, rehabilitación de infractores de ley y su reinserción social, en la forma que establezca la ley y dentro del marco de la política nacional de seguridad pública interior.

Además, SENDA está encargado de administrar el fondo establecido por el artículo 46 de la Ley No 20.000, que sanciona el tráfico ilícito de estupefacientes y sustancias sicotrópicas (Secretaría de Prevención del Delito, 2011).

SENDA es un servicio público, que se crea en un Ministerio orientado a la persecución del delito y control del consumo y tráfico de drogas, siendo esta su principal función como política pública. Observa a una persona joven, en etapa escolar (algunos en situación de desescolarización), con policonsumo de sustancias, como un potencial riesgo para la comunidad, instalándo- 
se así un imaginario de estigmatización social de delincuencia para toda la juventud con dichas prácticas, sin abordar esta temática como un problema que necesita una mirada global para su comprensión, es decir, con una perspectiva de atención integral más que punitivo, en especial a la población joven de los sectores más vulnerables del país. "La asociación droga y delito es una construcción social. Cuando la prohibición inventó la asociación droga $=$ delito empezó a operar para los fines prácticos como si fuera una asociación auténticamente real" (Vivanco, 2015, p. 77).

Si bien existen programas creados por SENDA, en cuanto a la atención ambulatoria, para jóvenes con consumo problemático de drogas, como el PAI (Programa Ambulatorio Intensivo), esta oferta está destinada exclusivamente a jóvenes que hayan cometido delito, mayores de 14 años y menores de 18, imputados o condenados bajo la Ley 20.084, que establece un sistema de responsabilidad de los adolescentes por infracciones a la ley penal (SENDA, 2011).

Claro ejemplo de aquello es que la política pública respecto del consumo abusivo de sustancias en jóvenes menores de 18 años, se encuentra radicado del Ministerio del Interior y Seguridad Pública y no en el Ministerio de Salud. Es urgente entonces que exista un sistema de protección integral, basado en contribuir a la calidad de vida y acceso a políticas sanitario-ambientales, que permitan el mejoramiento sostenido de la salud de la población juvenil, especialmente de los sectores más pobres. Todas las entidades de los servicios públicos, como educación, salud, protección, necesitan de un sistema de coordinación intersectorial, con el fin de entregar una atención integral, esto es, que sea una obligación legal para las instituciones públicas proteger los derechos de la juventud y la niñez.

Si bien en los últimos años ha existido un esfuerzo por parte de los gobiernos de turno en mejorar la atención integral intersectorial hacia la niñez y juventud, con la creación de Ordinarios y Decre- 
tos desde SENAME ${ }^{9}$, en conjunto con el Ministerio de Justicia y Derechos Humanos, Ministerio de Salud y SENDA ${ }^{10}$, estas medidas resultan insuficientes para la atención global de la población mencionada, ya que funcionan actualmente como programas piloto en algunas comunas del país, sin ser una política de Estado, ya que Chile no cuenta con una ley garante de derechos hacia la niñez y juventud que obligue a su cumplimento.

Por este escenario, el Comité de los Derechos del Niños de las Naciones Unidas (2007) ha observado en más de una ocasión a nuestro país, indicando:

El Comité celebra la incorporación de numerosos artículos sobre los derechos del niño en la Constitución y en numerosas enmiendas legislativas. No obstante, el Comité lamenta que todavía no se haya ultimado la reforma de la Ley de menores (Ley No 16.618 de 1967), con el fin de contar con una ley integral de protección del menor, según lo recomendado por el Comité en sus observaciones finales anteriores de 2002 (CRC/C/15/Add.173)... El Comité reitera su preocupación a este respecto y recomienda al Estado Parte que ultime con celeridad el proceso de reforma de la Ley de menores de 1967, a fin de brindar una protección integral a todos los niños. El Comité recomienda además que se haga una distinción clara, en los procedimientos judiciales y a todos los demás efectos, entre los niños que necesitan protección y aquellos que han entrado en conflicto con la ley. (UNICEF, 2007, p. 3)

9 El Servicio Nacional de Menores es un organismo gubernamental centralizado, colaborador del sistema judicial y dependiente del Ministerio de Justicia y Derechos Humanos.

10 Ord. N ${ }^{\circ}$ 183-18/01/17: Atención Prioritaria de NNA en Centros de Especialidad; Ord. $N^{\circ} 785-16 / 02 / 18$ : Orientaciones Técnicas y Administrativas de Salud en la APS de NNAJ que participan en los Programas del SENAME; Ord. N 425 (26/01/18) Lineamientos para la puesta en marcha del Sistema Intersectorial; Decreto Exento $\mathrm{N}^{\circ}$ 390-19/02/18: Aprueba Convenio de Colaboración sobre transferencia de datos entre MINJU, MINSAL y SENAME; Orientaciones Técnicas Chile Crece Contigo para la atención de niños y niñas de los Programas del SENAME). 
En Chile, cuando un joven en etapa escolar presenta alto compromiso biopsicosocial, debido al consumo problemático de alcohol y otras drogas (sumado al total abandono familiar), no puede acceder a un proceso de tratamiento para su rehabilitación, ya que no existe una oferta programática de los servicios públicos que entregue dicha atención.

Los programas ambulatorios y residencias que ofrece SENAME, no cuentan con dichas especialidades dentro de sus líneas técnicas. Tampoco existe de parte de la política de salud (desde el Ministerio de Salud) la disposición a atender esta problemática, a excepción de casos en los jóvenes menores de 18 años se encuentran en un estado de alto deterioro en su funcionamiento biopsicosocial, es decir, con alto riesgo vital. La atención en estos casos es para la desintoxicación del joven en Unidad de Corta Estadía o $\mathrm{UDAC}^{11}$ de los hospitales públicos, los cuales poseen una lista de espera por escasez de camas (el Hospital Calvo Mackenna ${ }^{12}$ cuenta solo con ocho camas) y especialistas. Cuando un joven logra ingresar y ser desintoxicado por la UDAC, una vez dada el alta médica, vuelve al mismo contexto social y grupo familiar, sin tener un seguimiento psicológico y psiquiátrico, por lo tanto es muy probable que las conductas de consumo y riesgo se repitan.

El articulo $\mathrm{N}^{\circ} 24$ de la Convención de los Derechos del Niño, Niña y Adolescente señala que:

1. Los Estados Partes reconocen el derecho del niño al disfrute del más alto nivel posible de salud y a servicios para el tratamiento de las enfermedades y la rehabilitación de la salud. Los

11 La Unidad de Desintoxicación y tratamiento para adolescentes con trastornos conductuales severos del Instituto Psiquiátrico Dr. José Horwitz Barak, es una unidad de corta estadía infantojuvenil, para adolescentes varones desde los 14 a 17 años 11 meses 30 días, infractores de ley condenados por tribunales de justicia en virtud de la Ley de Responsabilidad Penal Juvenil (Ley 20.084), a la sanción accesoria de rehabilitación de alcohol/drogas o bien con medidas cautelares o suspensión condicional que contempla esta rehabilitación. Más información en https://www. psiquiatrico.cl/index.php/117-udac

12 Más información en http://www.calvomackenna.cl/nuestros-servicios/atencionhospitalizados.php 
Estados Partes se esforzarán por asegurar que ningún niño sea privado de su derecho al disfrute de esos servicios sanitarios... 3. Los Estados Partes adoptarán todas las medidas eficaces y apropiadas posibles para abolir las prácticas tradicionales que sean perjudiciales para la salud de los niños... Salud y servicios médicos: Los niños tienen derecho a disfrutar del más alto nivel posible de salud y tener acceso a servicios médicos y de rehabilitación, con especial énfasis en aquellos relacionados con la atención primaria de salud, los cuidados preventivos y la disminución de la mortalidad infantil. Es obligación del Estado tomar las medidas necesarias, orientadas a la abolición de las prácticas tradicionales perjudiciales para la salud del niño. (Asamblea General de la ONU, 1989, pp. 36-37)

Contribuir a la promoción, protección y restitución de derechos de niños, niñas y adolescentes vulnerados, así como a la responsabilización y reinserción social de los jóvenes menores de 18 años con riesgo vital por consumo abusivo de drogas, a través de programas ejecutados directamente o por organismos colaboradores, es un trabajo y compromiso que ratificó Chile en la Convención citada. Sin embargo, no ha cumplido con ello, teniendo la urgencia y necesidad de abordarlo como tema prioritario, ante los últimos informes que dan cuentan de las altas vulneraciones que, desde la política de niñez, se han cometido (Informe IN$\mathrm{DH}^{13}$, 2007).

\section{Conclusiones}

Las formas de concebir a la juventud empobrecida desde la política pública de Chile (menores de 18 años y pertenecientes a sectores vulnerables), es a través de miradas tales como delincuencia, deserción escolar y consumo o tráfico de drogas. Es decir, se abor-

\footnotetext{
Instituto Nacional de Derechos Humanos, Diagnóstico de la situación de derechos humanos de niños, niñas y adolescentes en centros de protección de la red SENAME (2017). Más información en https://www.indh.cl/destacados-2/mision-deobservacion-sename-2017/
} 
da a la adolescencia y juventud empobrecida desde una perspectiva de riesgo o peligro en lo relativo a la constitución de una identidad sana, por lo tanto, se sanciona en vez de entregar una oferta o servicio de protección integral.

Las medidas actuales para abordar los temas asociados con el consumo de sustancias en jóvenes, tienden a tomar un enfoque que delega la responsabilidad a las personas, o sea, se aborda la problemática desde el castigo, desplegando una limitada contextualización de lo que empuja a un niño, niña o joven a encontrase en un estado de daño en el ámbito de su salud, y abandonado por sus figuras de cuidado, como también del sistémico abandono social en el cual está inserto.

Esto hace alusión a la nula posibilidad de contar con una oferta programática desde los servicios públicos actuales, que pudiera brindar apoyo y soporte proteccional hacia dicha población en situación de alto riesgo, ya que, como se ha referido en el presente estudio, la sociedad y todo su conjunto impacta fuertemente en el desarrollo biopsicosocial y la posibilidad que cada persona experimenta dentro de su ciclo vital.

Estas formas y servicios para enfrentar las temáticas que afectan a la juventud en etapa escolar, o sea, menores de edad, se encuentran enmarcadas en un modelo económico y estilo de vida individualista, al estar insertos bajo componentes de una superestructura de carácter neoliberal, que no tiene concordancia con el contexto que vive una población empobrecida.

Existe evidencia de que los altos niveles de desigualdad pueden generar deterioro en la cohesión social, un menor potencial de crecimiento económico o un descontento respecto de cómo se distribuyen los frutos de dicho crecimiento. Los fenómenos de la pobreza y la desigualdad han traído como consecuencia una fuerte dependencia de origen, esto significa que el lugar de nacimiento de las personas determina, en gran parte, las oportunidades que tendrán para surgir y desarrollarse plenamente. 
Asimismo, el abordaje de los servicios sociales hacia la juventud con consumo de sustancias, ya sean servicios proteccionales, de salud o educación, son insuficientes para la compleja realidad social que viven algunos adolescentes de familias empobrecidas, quienes experimentan compromiso biopsicosocial por el uso abusivo de drogas, con un alto riesgo vital. Ello a pesar de que estos jóvenes fueron observados por toda la red de infancia comunal (programas de intervención especializada), sin que los profesionales de las instituciones intervinientes consiguieran hacer efectiva una protección integral, ya que Chile no cuenta con una ley que la garantice, ocurriendo así, en algunos casos, el fallecimiento de estos jóvenes.

La realidad descrita permite apreciar el frágil trabajo en la articulación actual de las redes comunales de protección especializada que existe en Chile cuando se presenta una situación de alto riesgo por consumo de drogas, y como esto afecta tanto al sujeto (joven) como al trabajo desarrollado por los programas psicosociales interventores, al verse estos limitados en la ejecución de intervenciones efectivas para detener el escenario vulneratorio. En este sentido, existe la urgencia y prioridad de exigir hoy una política de protección integral hacia la niñez y juventud, la cual se ha tramitado por los gobiernos de turno.

Al presente, Chile no cuenta con un Sistema de Garantías de los Derechos de la Niñez, aunque hace poco fue ingresado a tramitación legislativa por el mensaje presidencial en septiembre de 2015. Este proyecto de ley se propone como una ley Marco que siente las bases generales. Ello supone el desarrollo de futuros cuerpos normativos, en los que aún se trabaja. Algunas de las iniciativas de ley ya han sido presentadas, como la creación de La Defensoría de los Derechos de la Niñez y La Subsecretaría de la Infancia, recién operando desde 2018, quedando pendiente los Servicios de Reinserción Social Juvenil y de Protección para niños, niñas y adolescentes. También está pendiente la modificación de diversos cuerpos legales, entre ellos, la Ley $\mathrm{N}^{\circ} 20.084$ sobre Responsabilidad Penal Adolescente; la Ley $\mathrm{N}^{\circ} 20.032$ de red de colaboradores del SENA- 
ME y Subvenciones, y la Ley $N^{\circ} 19.968$ de Tribunales de Familia (Biblioteca del Congreso Nacional de Chile, 2017).

Ante este escenario, la ley Marco resulta poca específica, ya que las garantías hacia la niñez y juventud aparecen débilmente consagradas en sus definiciones, mal diseñadas o incompletas en aspectos fundamentales, además de no detallar la forma en que estas garantías pueden ser exigidas.

Otro punto importante es que la ley Marco queda sujeta a la subordinación presupuestaria, es decir, condiciona el ejercicio de los derechos de niñez y juventud a los recursos de que disponga cada gobierno, dejando así esto a la voluntad y disposición de los legisladores.

Por consiguiente, es necesario generar mayor voluntad política para garantizar, de forma legal, los derechos humanos básicos hacia la juventud y la niñez, como también la transformación cultural de la sociedad que habitamos, a fin de hacernos conscientes de la responsabilidad de cada individuo en la convivencia con otros y con nuestro entorno, y que la mirada desde la política pública no sea punitiva, sino más bien un derecho básico para la comunidad.

\section{Bibliografía}

Asamblea General de la ONU. (1989). Convención Sobre los Derechos del Niño. Santiago de Chile: ACHNU Ediciones.

Bendit, R. y Stokes, D. (2004). Jóvenes en situación de desventaja social: políticas de transición entre la construcción social y las necesidades de una juventud vulnerable. Recuperado el 16 de octubre de 2018 de file://C:/Users/admin/Downloads/jovenesendesventaja7.pdf

Biblioteca del Congreso Nacional de Chile. (2009). Declaración de los Derechos Humanos de 1948. Recuperado el 28 de mayo de 2018 de https://www.leychile.cl/Navegar?idNorma=1000396

Biblioteca del Congreso Nacional de Chile. (2017). Sistema de Garantías de Derechos de la Niñez: Proyecto Informado por la Comisión de Familia y Adulto Mayor. Recuperado el 12 de septiembre de 2018 de https://www.bcn.cl/obtienearchivo?i d=repositorio/10221/23993/2/BCN_Proyecto\%20Garantias\%20Infancia\%20Familia.pdf 
Biblioteca Digital del Instituto Nacional de los Derechos Humanos. (2014). Declaración Universal de los Derechos Humanos 1948. Recuperado el 12 de septiembre de 2018 de https://bibliotecadigital.indh.cl/bitstream/handle/123456789/903/ declaracion-der.pdf?sequence $=1$

Bourdieu, P. (1990). La juventud no es más que una palabra. Recuperado el 13 de abril de 2018 de https://periferiaactiva.files.wordpress.com/2018/03/bourdieu-p-1990-e2809cla-juventudno-es-mc3a1s-que-una-palabrae2809d.pdf

Comellas, M. (1996). Familias Multiproblemáticas: La paradoja de la sociedad del bienestar. Recuperado el 30 de junio de 2018 de https://www.raco.cat/index.php/EducacioSocial/article/ viewFile/142325/384912

Cuevas, H. (2015). Precariedad, Precariado y Precarización: Un comentario crítico desde América Latina. Recuperado el 20 de octubre de 2018 de https://scielo.conicyt.cl/pdf/polis/ v14n40/art15.pdf

Chile Crece Contigo. (2015). Manual talleres de promoción temprana del desarrollo motor y lenguaje $n$ el primer año de vida. Recuperado el 2 de noviembre de 2018 de http://www.crececontigo.gob.cl/wp-content/uploads/2016/04/Manual-Taller-promocion-desarrollo-lenguaje-y-motor.pdf

Duarte, K. (2000). ¿juventud o juventudes? Versiones, trampas, pistas y ejes para acercarnos progresivamente a los mundos juveniles. Recuperado el 5 de mayo de 2018 de http:// repositorio.uchile.cl/bitstream/handle/2250/121857/ Juventud_o_juventudes_versiones.pdf?sequence $=1$

Duarte, K. (2012). Sociedades adultocéntricas: sobre sus orígenes y reproducción. Recuperado el 20 de julio de 2018 de https://scielo.conicyt.cl/pdf/udecada/v20n36/art05.pdf

Encuesta CASEN. (2015). ¿Qué es la encuesta CASEN? Recuperado el 4 de octubre de 2018 de http://encuestacasen.cl/

Fernández, M. (2012). El costo socioeconómico del consumo de drogas ilícitas en Chile. Recuperado el 13 de septiembre de 2018 de https://repositorio.cepal. org/bitstream/handle/11362/11550/107093114_ es.pdf? sequence $=1$ \&isAllowed $=y$

Fondo de las Naciones Unidas para la Infancia. (1990). Herramientas para la participación adolescente. Recuperado el 14 de noviembre de 2018 de http://www.herramientasparticipacion.edu.uy/index.php?option=com_content\&view=artic le\&id $=271 \&$ catid $=93 \&$ Itemid $=344$ 
Fondo de las Naciones Unidas para la Infancia. (2007). Observaciones del Comité a Chile: Informe Comité 2007, $44^{\circ}$ periodo de sesiones. Recuperado el 16 de octubre de 2018 de http://unicef. cl/web/observaciones-del-comite-a-chile/

Fondo de las Naciones Unidas para la Infancia. (1990). ¿Qué es la Convención sobre los Derechos del Niño? Recuperado el 2 de diciembre de 2018 de https://www.unicef.org/spanish/crc/index_30229.html

Gómez, E. (2007). Familias Multiproblemáticas y en Riesgo Social: Características e Intervención. Recuperado el 28 de mayo de 2018 de https://scielo.conicyt.cl/scielo.php?script=sci_artt ext\&pid=S0718-22282007000200004

Instituto Nacional de Derechos Humanos. (2017). Diagnóstico de la situación de derechos humanos de niños, niñas y adolescentes en centros de protección de la red SENAME. Recuperado el 20 de octubre de 2018 de https://www.indh.cl/destacados-2/mision-de-observacion-sename-2017/

Ministerio de Salud. (2011). Orientaciones técnicas para el control de salud integral de adolescentes. Recuperado el 29 de septiembre de 2018 de http://www.bibliotecaminsal.cl/wp/wpcontent/uploads/2016/04/GUIA-CLINICA-CONSUMOALCOHOL-MENORES-20-IMPRESA.pdf

Ministerio de Desarrollo Social. (2017). Informe de Desarrollo Social 2017. Recuperado el 20 de junio de 201 de http://www.ministeriodesarrollosocial.gob.cl/pdf/upload/IDS2017.pdf

Ministerio de Desarrollo Social. (2011). Informe de Política Social. Recuperado el 15 de junio de 2018 de http://www.ministeriodesarrollosocial.gob.cl/ipos/pdf/ipos_2011_pobreza_desigualdad_oportunidades.pdf

Organización Mundial de la Salud. (2018). Desarrollo en la adolescencia. Recuperado el 10 de octubre de 2018 de https://www. who.int/maternal_child_adolescent/topics/adolescence/ dev/es/

Pizarro, R. (2001). La Vulnerabilidad social y sus desafíos: una mirada dese América Latina. Recuperado el 4 de octubre de 2018 de https://repositorio.cepal.org/bitstream/handle/11362/4762/S0102116_es.pdf

Rodríguez, J. (2001). Vulnerabilidad y grupos vulnerables: un marco de referencia conceptual mirando a los jóvenes. Recuperado el 18 de abril de 2018 de https://repositorio.cepal.org/bitstream/handle/11362/7150/S018659_es.pdf?sequence $=1$

Rojas, G., Gaete, J., Guajardo, V., Martínez, V., Barroilhet, S., Meneses, J., Fritsh, R., Araya, R. (2012). Consumo de sustancias y sinto- 
mas depresivos en escolares de liceos municipales de Santiago. Rev Med Chile, 140, 184-191.

Servicio Nacional de Menores. (2015). Bases y orientaciones técnicas: línea de acción oficinas de protección de derechos de niños, niña y adolescentes. Recuperado el 1 de octubre de 2018 de http://www.sename.cl/wsename/p13_15-11-2018/BasesOrientaciones-Tecnicas-OPD.pdf

Servicio Nacional para la Prevención y Rehabilitación del Consumo de Drogas y Alcohol. (2011). Funciones. Recuperado el 28 de octubre de 2018 de http://www.senda.gob.cl/quienes-somos/funciones/

Servicio Nacional para la Prevención y Rehabilitación del Consumo de Drogas y Alcohol. (2017). Informativo SENDA. Recuperado el 28 de octubre de 2018 de http://www.senda.gob.cl/ wp-content/uploads/2018/01/InformativoSENDA2017. pdf

Subsecretaria de Prevención de Delito. (2011). Funciones: Ministerio del Interior y de Seguridad Publica. Recuperado el 10 de octubre de 2018 desde http://www.seguridadpublica.gov.cl/ nosotros/

Vivanco, M. (2015). Críticas a la moral conservadora. Santiago, Chile: LOM Ediciones. 